\title{
Resonance in orbits of plane partitions
}

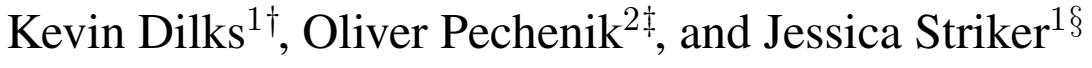 \\ ${ }^{1}$ Department of Mathematics, North Dakota State University, Fargo, ND 58102 USA \\ ${ }^{2}$ Department of Mathematics, University of Illinois at Urbana-Champaign, Urbana, IL 61801 USA
}

\begin{abstract}
We introduce a new concept of resonance on discrete dynamical systems. Our main result is an equivariant bijection between plane partitions in a box under rowmotion and increasing tableaux under $K$-promotion, using a generalization of the equivariance of promotion and rowmotion [J. Striker-N. Williams '12] to higher dimensional lattices. This theorem implies new results for $K$-promotion and new proofs of previous results on plane partitions.

Résumé. Nous introduisons le nouveau concept de résonance pour les systèmes dynamiques discrets. Notre résultat principal est une bijection équivariante entre partitions planes dans une boîte sous rowmotion et tableaux croissants sous $K$-promotion, en utilisant une généralisation de la équivariance de promotion et de rowmotion [J. StrikerN. Williams '12] aux treillis en dimensions supérieures. Ce théorème implique de nouveaux résultats pour $K$ promotion et de nouvelles preuves de résultats connus pour partitions planes.
\end{abstract}

Keywords. resonance, increasing tableaux, plane partitions, dynamical algebraic combinatorics

\section{Resonance}

We introduce the following concept of resonance(i)

Definition 1.1 Suppose $G=\langle g\rangle$ is a cyclic group acting on a set $X, \mathcal{C}_{\omega}=\langle c\rangle$ a cyclic group of order $\omega$ acting nontrivially on a set $Y$, and $f: X \rightarrow Y$ a surjection. We say the triple $(X, G, f)$ exhibits resonance with frequency $\omega$ if, for all $x \in X, c \cdot f(x)=f(g \cdot x)$.

In our examples, $Y$ will be either a set of combinatorial objects drawn in the plane with $c$ acting by rotation or a set of words with $c$ acting by a cyclic shift. Resonance is a pseudo-periodicity property of the $G$-action, in that the resonant frequency $\omega$ is generally less than the order of the $G$-action. Note that, in general, $\left(X, G, \operatorname{id}_{X}\right)$ satisfies the definition of resonance with frequency $|G|$; we call this an instance of trivial resonance.

\footnotetext{
${ }^{\dagger}$ Email: kevin.dilks@ndsu.edu.

‡Email: pecheni2@illinois.edu.

§Email: jessica.striker@ndsu.edu.

(i) The mathematically precise definition of resonance given here is new, though the phenomenon has been discussed by various people over the past year or more, in particular, at the 2015 "Dynamical Algebraic Combinatorics" workshop at the American Institute of Mathematics where work on this paper began. Thanks to J. Propp for coining the term "resonance" which so nicely encapsulates the idea.
}

1365-8050 @ 2016 Discrete Mathematics and Theoretical Computer Science (DMTCS), Nancy, France 
Our prototypical example of resonance is the action of gyration on alternating sign matrices. Alternating sign matrices are known to be in bijection with fully-packed loop configurations [Wi00, $\operatorname{Pr} 01]$. There is a (non-injective) map from a fully-packed loop configuration to its link pattern and an invertible action on fully-packed loops called gyration. We discuss these objects and maps in detail in [DPS16]; see Figure 1 for an example. The following gives a remarkable property of gyration that we reformulate into a statement of resonance.

Theorem 1.2 (B. Wieland [Wi00|) Gyration of a fully-packed loop configuration rotates the associated link pattern by an angle of $2 \pi / 2 n$.

Corollary 1.3 Let $\mathrm{ASM}_{n}$ be the set of $n \times n$ alternating sign matrices, $f$ be the map from an alternating sign matrix through its fully-packed loop configuration to the link pattern, and Gyr be gyration. Then $\left(\mathrm{ASM}_{n},\langle\mathrm{Gyr}\rangle, f\right)$ exhibits resonance with frequency $2 n$.

For example, consider gyration on $5 \times 5$ alternating sign matrices. Gyration has orbits of size 2, 4, 5, and 10. So the order of gyration in this case is 20 , but $\left(\mathrm{ASM}_{5}, \mathrm{Gyr}, f\right)$ exhibits resonance with frequency 10. Consider the orbit of gyration in Figure 1. This orbit is of size 4, while the link pattern orbit is of size 2. So even though $\operatorname{Gyr}^{10}(A) \neq A, \operatorname{rot}^{10}(f(A))=f(A)\left(\right.$ since $\operatorname{rot}^{2}(f(A))=f(A)$ ).

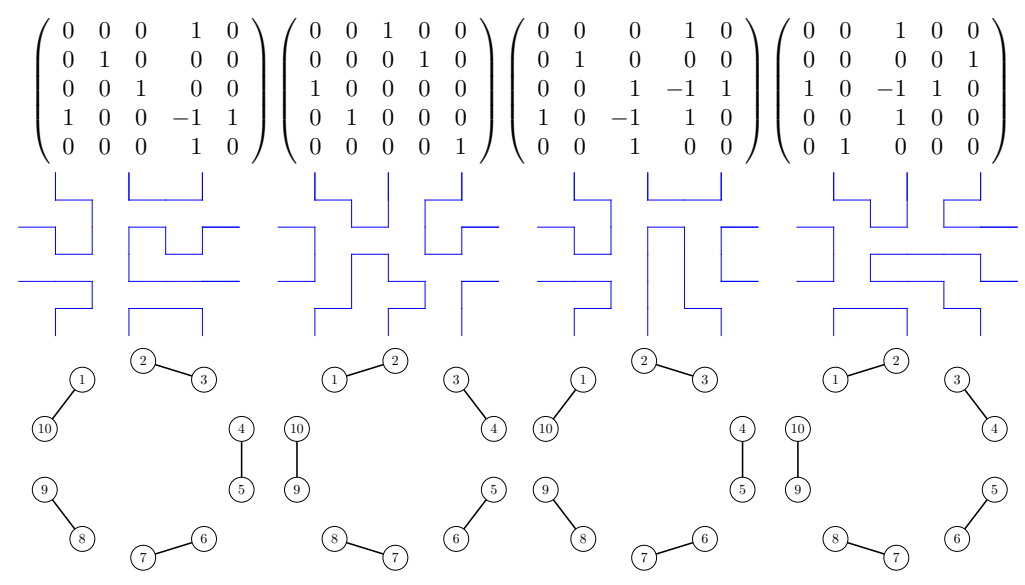

Fig. 1: A length 4 gyration orbit in $A S M_{5}$; Top Row: alternating sign matrices, Middle Row: the corresponding fully-packed loop configurations, Bottom Row: the associated link patterns

We think of the property of resonance as somewhat analogous to the cyclic sieving phenomenon (introduced by V. Reiner-D. Stanton-D. White [RSW04]) and the homomesy property (isolated by J. ProppT. Roby [PrRo15]) in being a rather subtle 'niceness' property of a cyclic group action. We suspect that the phenomenon of resonance, like those of cyclic sieving and homomesy, is significantly more common than previously realized. Heuristically, one is led to suspect the presence of resonance in a system by observing that many orbit cardinalities are multiples or divisors (or multiples of divisors) of $\omega$.

The rest of this paper is structured as follows. In Section 2, we recall the $K$-promotion operator on increasing tableaux and establish a number of new properties (including resonance) that we will use. In Section 3. we establish resonance of plane partitions under rowmotion, and extend machinery developed 
by N. Williams and the third author [StWi12], to introduce the family of toggle group actions $\left\{\mathrm{Pro}_{\pi, v}^{\sigma}\right\}$ and show that each $\operatorname{Pro}_{\pi, v}^{\sigma}$ acts with the same cycle structure as rowmotion. In Section 4 , we give an equivariant bijection between increasing tableaux under $K$-promotion and plane partitions under $\operatorname{Pro}_{(1,1,-1)}$ and Row. We then extract a number of corollaries from this equivariant bijection, including new proofs of theorems of A. Brouwer-A. Schrijver [BrSc74], a strengthening of a theorem of P. Cameron-D. Fon-derFlaass [CaFo95], and several new results on the order of $K$-promotion. Finally, we conjecture the order of rowmotion on plane partitions of height 3 , as well several additional instances of resonance related to alternating sign matrices and totally symmetric self-complementary plane partitions.

This document is an extended abstract; for full details, see the version available on the arXiv [DPS16].

\section{$2 K$-Promotion on increasing tableaux}

In this section, we study increasing tableaux, the first of the objects in our main bijection (Theorem 4.1]. After recalling the basic concepts, we establish resonance of increasing tableaux under $K$-promotion in Theorem 2.2. In Section 2.3, we reinterpret $K$-promotion in terms of $K$-Bender-Knuth involutions, which we introduce; this will be important in Section 4.2 in establishing equivariance of our main bijection.

\subsection{Increasing tableaux}

Identify a partition $\lambda$ with its Young diagram. An increasing tableau of shape $\lambda$ is a filling of this Young diagram with positive integers with the property that labels strictly increase from left to right across rows and from top to bottom down columns. An example appears in Figure 3 . We write $\operatorname{Inc}^{q}(\lambda)$ for the set of all increasing tableaux of shape $\lambda$ with all entries at most $q$. (In contrast to other definitions that have appeared in the literature, we do not assume that every integer between 1 and $q$ appears.)

Increasing tableaux have appeared in various contexts within algebraic combinatorics. Most notably for our purposes, H. Thomas-A. Yong introduced [ThYo09] a $K$-jeu de taquin algorithm for increasing tableaux, which they applied to $K$-theoretic Schubert calculus, obtaining Littlewood-Richardson rules for the Grothendieck rings of algebraic vector bundles over Grassmannians. In [Pe14], the second author studied a $K$-promotion operator, analogous to that of M.-P. Schützenberger [Sc72] for semistandard tableaux, but using $K$-jeu de taquin in place of ordinary jeu de taquin. $K$-promotion has been further studied by J. Bloom-D. Saracino and the second author [BPS16], T. Pressey-A. Stokke-T. Visentin [PSV14] and B. Rhoades [Rh15].

$K$-promotion is defined as follows. Let $T \in \operatorname{Inc}^{q}(\lambda)$. Delete all labels 1 from $T$. (Note there is at most one such label.) Consider the set of boxes that are either empty or contain 2 . This set naturally decomposes into connected components that are short ribbons, i.e. connected skew shapes containing no $2 \times 2$ subshape and with each column and row of length at most 2 . For each such short ribbon containing more than one box, we delete each label 2 , while simultaneously placing 2 in each empty box. We do not make any change to short ribbons consisting of a single box. Now consider the set of boxes that are either empty or contain 3, and repeat the above process. Continue until all empty boxes are located at outer corners of $\lambda$. Finally, label those boxes $q+1$ and then subtract 1 from every entry. The result is $K-\operatorname{Pro}(T) \in \operatorname{Inc}^{q}(\lambda)$.

\subsection{Content cycling}

Define the content of an increasing tableau $T \in \operatorname{Inc}^{q}(\lambda)$ to be the sequence $\operatorname{Con}(T)=\left(a_{1}, a_{2}, \ldots, a_{q}\right)$, where $a_{i}=1$ if $i$ is an entry of $T$ and $a_{i}=0$ if it is not. That is, $a_{i}:=\chi_{i}(T)$, where $\chi_{i}$ denotes the 


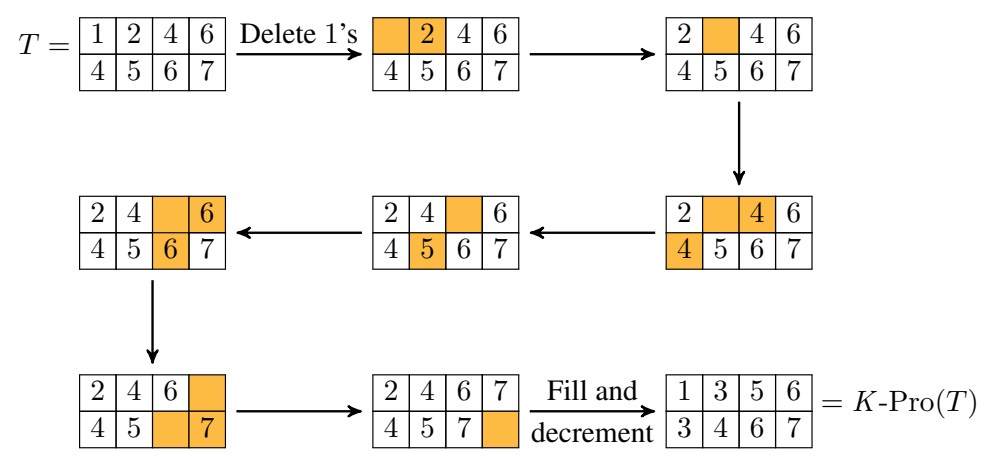

Fig. 2: Calculating the $K$-promotion of $T \in \operatorname{Inc}^{7}(2 \times 4)$. In each intermediate step, we have colored the short ribbons on which we are about to act.

indicator function for the label $i$.

Lemma 2.1 Let $T \in \operatorname{Inc}^{q}(\lambda)$. If $\operatorname{Con}(T)=\left(a_{1}, a_{2}, \ldots, a_{q}\right)$, then $\operatorname{Con}(K-\operatorname{Pro}(T))$ is the cyclic shift $\left(a_{2}, \ldots, a_{q}, a_{1}\right)$.

The following instance of resonance follows directly from Lemma 2.1

Theorem $2.2\left(\operatorname{Inc}^{q}(\lambda),\langle K\right.$-Pro $\rangle$, Con) exhibits resonance with frequency $q$.

This leads to the following corollary.

Corollary 2.3 Suppose $q$ is prime and $T \in \operatorname{Inc}^{q}(\lambda)$ does not have full content. Then the size of the $K$-promotion orbit of $T$ is a multiple of $q$.

\section{$2.3 K$-promotion in terms of $K$-Bender-Knuth involutions}

In this subsection, we reinterpret $K$-promotion as a product of involutions, which we will need in our proof of Theorem 4.4. We define operators $K-\mathrm{BK}_{i}$ on $\operatorname{Inc}^{q}(\lambda)$ for each $1 \leq i \leq q$. Take $T \in \operatorname{Inc}^{q}(\lambda)$. We compute $K-\mathrm{BK}_{i}(T)$ as follows: Consider the set of boxes in $T$ that contain either $i$ or $i+1$. This set decomposes into connected components that are short ribbons. On each nontrivial such component, we do nothing. On each component that is a single box, replace the symbol $i$ by $i+1$ or vice versa. The result is $K-\mathrm{BK}_{i}(T)$. That is, the action of $K-\mathrm{BK}_{i}$ on $T$ is to increment $i$ and/or decrement $i+1$, wherever possible. These involutions are illustrated in Figure 3

Clearly each $K-\mathrm{BK}_{i}$ is an involution. We call it the $i$ th $K$-Bender-Knuth involution because in the case $T$ is standard, $K-\mathrm{BK}_{i}$ coincides with the classical involution introduced by E. Bender-D. Knuth [BeKn72].

Proposition 2.4 For $T \in \operatorname{Inc}^{q}(\lambda), K-\operatorname{Pro}(T)=K-\mathrm{BK}_{q-1} \circ \cdots \circ K-\mathrm{BK}_{1}(T)$.

Proof: Another way to think of $K-\mathrm{BK}_{i}$ is as the $K$-infusion [ThYo09] of the labels $i$ through the labels $i+1$. That is, treat the labels $i$ as empty boxes and swap the short ribbons of empty boxes and $(i+1)$ 's as in the definition of $K$-promotion; then relabel each $i+1$ as $i$ and each empty box as $i+1$. 


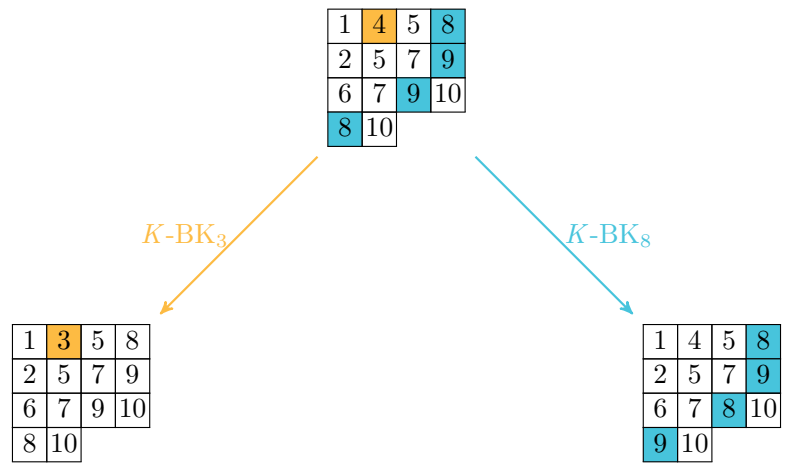

Fig. 3: The action of some K-Bender-Knuth involutions on an increasing tableau $T$ of shape $(4,4,4,2)$.

From this characterization, one sees $K-\mathrm{BK}_{q-1} \circ \cdots \circ K-\mathrm{BK}_{1}$ amounts to deleting the 1 's and swapping the empty boxes through all other labels in order, decrementing each other label as the empty boxes swap through it, and finally labeling the empty boxes at outer corners by $q$. This is the same as $K$-promotion, except that the decrementing of labels happens throughout the process instead of all at the end.

\section{Promotion and rowmotion, revisited}

In this section, we switch our focus from increasing tableaux to our other main objects of study: plane partitions. A plane partition is a stack of unit cubes in the positive orthant, justified toward the origin in all three directions. Plane partitions inside an $a \times b \times c$ box can be seen as order ideals in the product of three chains poset $\mathbf{a} \times \mathbf{b} \times \mathbf{c}$. Thus, most of our discussion in this section centers on posets and order ideals, keeping in mind that all such general results can be applied to plane partitions.

\subsection{Rowmotion}

Let $P$ be a finite partially ordered set (poset). $P$ is a chain if all its elements are mutually comparable. Let $\mathbf{n}$ denote the $n$-element chain. The product of $k$ chains poset, $P=\mathbf{n}_{\mathbf{1}} \times \mathbf{n}_{\mathbf{2}} \times \cdots \mathbf{n}_{\mathbf{k}}$, has as elements ordered integer $k$-tuples $\left(x_{1}, x_{2}, \ldots, x_{k}\right)$ such that $0 \leq x_{i} \leq n_{i}-1$ with partial order given by componentwise comparison. A subset $I \subseteq P$ is an order ideal if it is closed downward, i.e. if $y \in I$ and $x \leq y$, then $x \in I$. Denote the set of order ideals of $P$ as $J(P)$.

We study the orbit structure of rowmotion, Row: $J(P) \rightarrow J(P)$, defined as the order ideal whose maximal elements are the minimal elements of $P \backslash I$; see [StWi12] for a detailed history. A. BrouwerA. Schrijver [BrSc74] studied Row for $P=\mathbf{a} \times \mathbf{b}$. They discovered:

Theorem 3.1 (A. Brouwer-A. Schrijver [BrSc74]) The order of Row on $J(\mathbf{a} \times \mathbf{b})$ is $a+b$.

P. Cameron-D. Fon-der-Flaass [CaFo95] studied the same question on plane partitions, that is, order ideals in the product of three chains $\mathbf{a} \times \mathbf{b} \times \mathbf{c}$.

Theorem 3.2 (P. Cameron-D. Fon-der-Flaass [CaFo95]) The order of Row on $J(\mathbf{a} \times \mathbf{b} \times \mathbf{2})$ is $a+b+1$. 
Extrapolating from Theorems 3.1 and 3.2, one might speculate that Row has order $a+b+c-1$ on $J(\mathbf{a} \times \mathbf{b} \times \mathbf{c})$. In general, the order is unknown but often significantly greater than this naive guess. However, P. Cameron-D. Fon-der-Flaass established the following.

Theorem 3.3 (P. Cameron-D. Fon-der-Flaass [CaFo95]) If $a+b+c-1$ is prime and $c>a b-a-b+1$, then the cardinality of every orbit of Row on $J(\mathbf{a} \times \mathbf{b} \times \mathbf{c})$ is a multiple of $a+b+c-1$.

We will revisit Theorems 3.1, 3.2, and 3.3 in Section 4.3

\subsection{The toggle group}

The toggle group, first studied by P. Cameron-D. Fon-der-Flaass [CaFo95], and later by N. Williams and the third author [StWi12], is the subgroup of the symmetric group on all order ideals $\mathfrak{S}_{J(P)}$ generated by certain involutions, called toggles. For each element $e \in P$ define its toggle $t_{e}: J(P) \rightarrow J(P)$ as

$$
t_{e}(I)= \begin{cases}I \cup\{e\} & \text { if } e \notin I \text { and } I \cup\{e\} \in J(P) \\ I \backslash\{e\} & \text { if } e \in I \text { and } I \backslash\{e\} \in J(P) \\ I & \text { otherwise }\end{cases}
$$

Remark 3.4 Observe that $t_{e}, t_{f}$ commute whenever neither e nor $f$ covers the other.

The following theorem interprets rowmotion as a toggle group action.

Theorem 3.5 ([CaFo95]) Given any poset $P$, Row is the toggle group element that toggles the elements in the reverse order of any linear extension. If $P$ is ranked, this is the same as toggling the ranks (rows) from top to bottom.

In 2012 [StWi12], N. Williams and the third author built on the work of P. Cameron-D. Fon-der-Flaass; they showed that rowmotion is conjugate to a toggle group element they called promotion, defined as toggling the elements of the poset from left to right (given a suitable notion of left-to-right, for which they used the term rc-poset). In Section 3.5 we generalize the conjugacy of promotion and rowmotion; first we use some machinery from [StWi12] to prove resonance of rowmotion on plane partitions.

\subsection{Resonance of plane partitions}

In this subsection, we give our second main resonance result, Theorem 3.8, which also yields a new proof of Theorem 3.3. For full details, see [DPS16].

In [StWi12, Section 7.2], N. Williams and the third author characterized $J(\mathbf{a} \times \mathbf{b} \times \mathbf{c})$ in terms of boundary path matrices, which are certain $b \times(a+b+c-1)$ matrices with entries in $\{0,1\}$. Promotion on the order ideal corresponds to a simple operation on the boundary path matrix (see [StWi12] for details).

Given $I \in J(\mathbf{a} \times \mathbf{b} \times \mathbf{c})$ with boundary path matrix $\left\{X_{i, j}\right\}$, define $X_{\max }(I)$ to be the vector of length $a+b+c-1$ whose $j$ th entry is $\max \left(X_{i, j}\right)_{1 \leq i \leq b}$.

Lemma 3.6 Let $I \in J(\mathbf{a} \times \mathbf{b} \times \mathbf{c})$. If $X_{\max }(I)=\left(x_{1}, x_{2}, \ldots, x_{a+b+c-1}\right)$, then $X_{\max }(\operatorname{Pro}(I))$ is the cyclic shift $\left(x_{2}, \ldots, x_{a+b+c-1}, x_{1}\right)$.

The following instance of resonance follows directly from Lemma 3.6

Proposition $3.7\left(J(\mathbf{a} \times \mathbf{b} \times \mathbf{c}),\langle\right.$ Pro $\left.\rangle, X_{\max }\right)$ exhibits resonance with frequency $a+b+c-1$.

By the equivariance of Pro and Row in [StWi12], we have the following statement of resonance. 
Theorem $3.8\left(J(\mathbf{a} \times \mathbf{b} \times \mathbf{c}),\langle\right.$ Row $\left.\rangle, X_{\max } \circ D\right)$ exhibits resonance with frequency $a+b+c-1$, where $D$ is the conjugating toggle group element between rowmotion and promotion given in [StWi12] Theorem 5.4].

This leads to the following corollary.

Corollary 3.9 Suppose $a+b+c-1$ is prime and $I \in J(\mathbf{a} \times \mathbf{b} \times \mathbf{c})$. Suppose there is a zero in $X_{\max }(I)$. Then the size of the promotion orbit of $I$ is a multiple of $a+b+c-1$.

Corollary 3.9 gives a new proof of Theorem 3.3 of P. Cameron-D. Fon-der-Flaass, as the condition $c>a b-a-b+1$ implies a zero in $X_{\max }(I)$, by the pigeonhole principle. Their original proof of Theorem 3.3 is somewhat more complicated, as it analyzes rowmotion directly.

\section{$3.4 n$-dimensional lattice projections}

In this and the next subsections, we adapt the proof of the conjugacy of promotion and rowmotion from [StWi12] to give a generalization in the setting of $n$-dimensional lattice projections, which we introduce in Definition 3.11. (This new perspective includes the original theorem as the case $n=2$.) We prove, in Theorem 3.19 , the equivariance of the $2^{n-1}$ toggle actions given in Definition 3.12

Definition 3.10 We say that a poset $P$ is ranked if it admits a rank function $\mathrm{rk}: P \rightarrow \mathbb{Z}$ satsifying $\operatorname{rk}(y)=\operatorname{rk}(x)+1$ whenever $y$ covers $x$.

Definition 3.11 We say that an (n-dimensional) lattice projection of a poset $P$ is an order and rank preserving map $\pi: P \rightarrow \mathbb{Z}^{n}$, where the rank function on $\mathbb{Z}^{n}$ is the sum of the coordinates and $x \leq y$ in $\mathbb{Z}^{n}$ if and only if the componentwise difference $y-x$ is in $\left(\mathbb{Z}_{\geq 0}\right)^{n}$.

In [StWi12], the definition of an rc-poset was a poset that had a 2-dimensional lattice projection (albeit to a slightly different lattice). However, E. Sawin noted (personal communication, 2013) that every ranked poset $P$ with rank function $\rho$ has such an embedding given by $\pi(x)=(\rho(x), 0)$ for $x \in P$. Similarly, any poset $P$ with a lattice projection $\pi$ has a rank function given by the sum of the coordinates in $\pi(x)$ for $x \in P$. Additionally, a ranked poset can have multiple distinct lattice projections. Therefore, instead of considering rc-posets, we consider any ranked poset, but with respect to a given lattice projection.

\subsection{Promotion via affine hyperplane toggles}

We now define a toggling order on our poset with respect to an $n$-dimensional lattice projection, and with respect to a distinguished direction.

Definition 3.12 Let $P$ be a poset with an $n$-dimensional lattice projection $\pi$, and choose a distinguished vector $v=\left(v_{1}, v_{2}, v_{3}, \ldots, v_{n}\right)$, where $v_{j} \in\{ \pm 1\}$. Let $T_{\pi, v}^{i}$ be the product of toggles $t_{x}$ for all elements $x$ of $P$ that lie on the affine hyperplane $\langle\pi(x), v\rangle=i$. If there is no such $x$, then this is the empty product, considered to be the identity. Then define promotion with respect to $\pi$ and $v$ as:

$$
\operatorname{Pro}_{\pi, v}=\ldots T_{\pi, v}^{-2} T_{\pi, v}^{-1} T_{\pi, v}^{0} T_{\pi, v}^{1} T_{\pi, v}^{2} \ldots
$$

Remark 3.13 Note that $\operatorname{Pro}_{\pi,-v}=\left(\mathrm{Pro}_{\pi, v}\right)^{-1}$, so we will generally only consider distinguished vectors with $v_{1}=1$, as all promotion operators are either of this form, or the inverse of something of this form.

Lemma 3.14 Two elements of the poset that lie on the same affine hyperplane $\langle\pi(x), v\rangle=i$ cannot be part of a covering relation, so by Remark 3.4 the operator $T_{\pi, v}^{i}$ is well-defined and $\left(T_{\pi, v}^{i}\right)^{2}=\mathrm{id}$. 

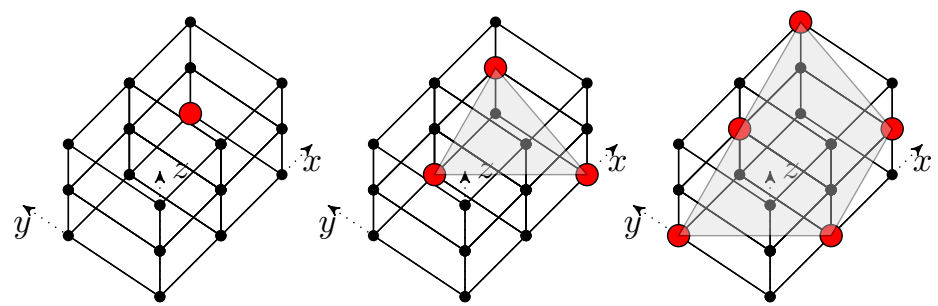

$x+y-z=3$

$x+y-z=2$

$x+y-z=1$
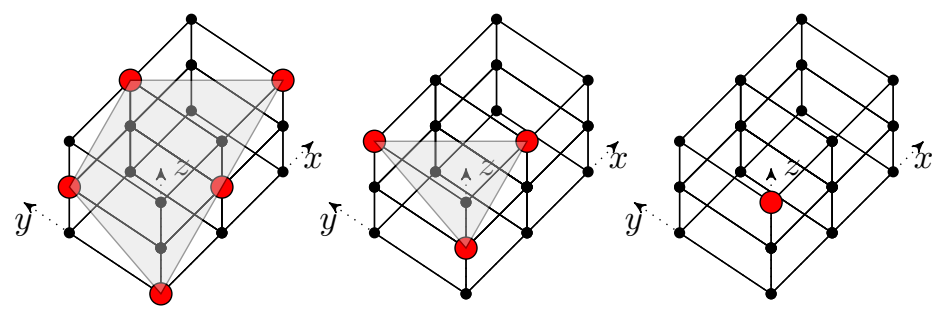

$$
x+y-z=0
$$

$$
x+y-z=-1
$$

$x+y-z=-2$

Fig. 4: The affine hyperplane toggles corresponding to $\operatorname{Pro}_{\mathrm{id},(1,1,-1)}$ for the identity three-dimensional lattice projection of the poset $J(\mathbf{3} \times \mathbf{2} \times \mathbf{3})$.

Note that for a finite poset $P, T_{\pi, v}^{i}$ will be the identity operator for all but finitely many $i$.

Remark 3.15 To compare with the notions of promotion and rowmotion given in [StWi12], for a given 2dimensional lattice projection $\pi$ of a finite poset $P$, rowmotion corresponds to $\operatorname{Pro}_{\pi,(1,1)}$, while promotion corresponds to $\operatorname{Pro}_{\pi,(1,-1)}$.

Proposition 3.16 For any finite ranked poset $P$ and lattice projection $\pi, \operatorname{Pro}_{\pi,(1,1, \ldots, 1)}=$ Row.

Proof: $\operatorname{Pro}_{\pi,(1,1, \ldots, 1)}$ sweeps through $P$ from top to bottom, so by Theorem 3.5 this is rowmotion.

We give some further definitions and lemmas, in order to state Theorem 3.19 in full generality.

Definition 3.17 Let $P$ be a poset, and let $\pi, v$, and $T_{\pi, v}^{i}$ be as in Definition 3.12. Define the support of $(P, \pi, v)$, denoted $\operatorname{supp}(P, \pi, v)$, to be the smallest interval $[a, b] \subseteq \mathbb{Z}$ such that $T_{\pi, v}^{i}$ is the identity operator for all $i \in \mathbb{Z} \backslash[a, b]$.

Definition 3.18 If $(P, \pi, v)$ has finite support, that is, $\operatorname{supp}(P, \pi, v)=[a, b] \subset \mathbb{Z}$, let $\sigma:[a, b] \rightarrow[a, b]$ be a bijective map. Then define promotion with respect to $P, \pi$, $v$, and $\sigma$ as the following product of hyperplane-toggles:

$$
\operatorname{Pro}_{\pi, v}^{\sigma}=T_{\pi, v}^{\sigma(a)} T_{\pi, v}^{\sigma(a+1)} \ldots T_{\pi, v}^{\sigma(b-1)} T_{\pi, v}^{\sigma(b)} .
$$

Theorem 3.19 Let $P$ be a finite poset with n-dimensional lattice projection $\pi$. Let $v=\left(v_{1}, v_{2}, \ldots, v_{n}\right)$ and $w=\left(w_{1}, w_{2}, \ldots, w_{n}\right)$, where $v_{j}, w_{j} \in\{ \pm 1\}$, and let $\sigma: \operatorname{supp}(P, \pi, v) \rightarrow \operatorname{supp}(P, \pi, v)$ and 
$\tau: \operatorname{supp}(P, \pi, w) \rightarrow \operatorname{supp}(P, \pi, w)$ be bijective maps. There is an equivariant bijection between $J(P)$ under $\operatorname{Pro}_{\pi, v}^{\sigma}$ and $J(P)$ under $\operatorname{Pro}_{\pi, w}^{\tau}$.

The proof of Theorem 3.19 follows the proof of [StWi12, Theorem 5.2]; see [DPS16] for details.

\section{An equivariant bijection between plane partitions and increas- ing tableaux}

\subsection{Bijections between increasing tableaux and plane partitions}

In this section, we introduce bijections between increasing tableaux and plane partitions. These bijections should not be at all surprising. However, these maps have amazing properties that will be key to many of our results. These maps are also fundamental to [HPPW16], where they are used to give the first bijective proofs of various results on plane partitions, including R. Proctor's main result from [Pr83].

We define a map $\Psi_{3}: J(\mathbf{a} \times \mathbf{b} \times \mathbf{c}) \rightarrow \operatorname{Inc}^{a+b+c-1}(a \times b)$ as follows. Let $I \in J(\mathbf{a} \times \mathbf{b} \times \mathbf{c})$. Thinking of $I$ in the standard way as a pile of small cubes in an $a \times b \times c$ box, project onto the $a \times b$ face. Record in position $(i, j)$ the number of boxes of $I$ with coordinate $(i, j, k)$ for some $0 \leq k \leq c-1$. The result is a standard plane partition representation of $X$, as a filling of the Young diagram $a \times b$ with nonnegative integers such that rows weakly decrease from left to right and columns weakly decrease from top to bottom. Rotate this plane partition $180^{\circ}$, so that rows and columns become weakly increasing. Now thinking of $a \times b$ as a graded poset with the upper left corner box the unique element of rank 0 , add to each label its rank plus 1 . That is, increase each label by one more than its distance from the upper left corner box. (This is just the standard way of converting a weakly increasing sequence into a strictly increasing one.) The result is the increasing tableau $\Psi_{3}(I)$. For an example, see Figure 5

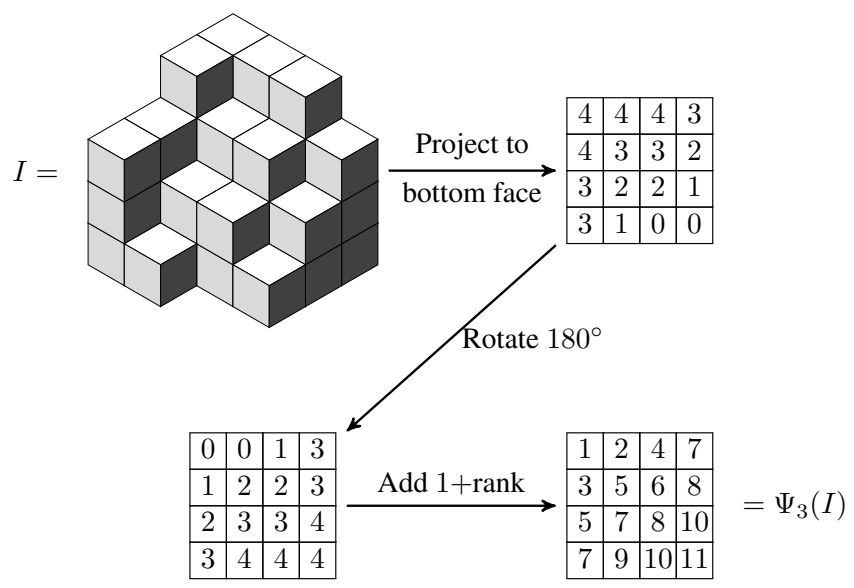

Fig. 5: The process of applying $\Psi_{3}$ to the illustrated $I \in J(\mathbf{4} \times \mathbf{4} \times \mathbf{4})$. Here we think of $\Psi_{3}$ as projecting onto the bottom face of the large containing box. 
Theorem 4.1 $\Psi_{3}: J(\mathbf{a} \times \mathbf{b} \times \mathbf{c}) \rightarrow \operatorname{Inc}^{a+b+c-1}(a \times b)$ gives a bijection between plane partitions inside an $a \times b \times c$ box and increasing tableaux of shape $a \times b$ and entries at most $a+b+c-1$.

Similarly, define bijections $\Psi_{2}: J(\mathbf{a} \times \mathbf{b} \times \mathbf{c}) \rightarrow \operatorname{Inc}^{a+b+c-1}(a \times c)$ and $\Psi_{1}: J(\mathbf{a} \times \mathbf{b} \times \mathbf{c}) \rightarrow$ Inc $^{a+b+c-1}(b \times c)$ projecting onto the $\mathbf{a} \times \mathbf{c}$ and $\mathbf{b} \times \mathbf{c}$ faces, respectively.

\subsection{The equivariance of $K$-Pro and Row}

Our first main result was Theorem 3.19, that given a poset $P$ and a lattice projection $\pi$, there is an equivariant bijection between $J(P)$ under $\operatorname{Pro}_{\pi, v}^{\sigma}$ and $J(P)$ under $\operatorname{Pro}_{\pi, w}^{\tau}$, where $\sigma, \tau$ are any permutations of the hyperplane toggles associated to the vectors $v, w$. In this section, we use Theorem 3.19 in the proof of our second main result, Theorem 4.4 , that $K$-Pro and Row are in equivariant bijection.

Lemma 4.2 The following diagram commutes:

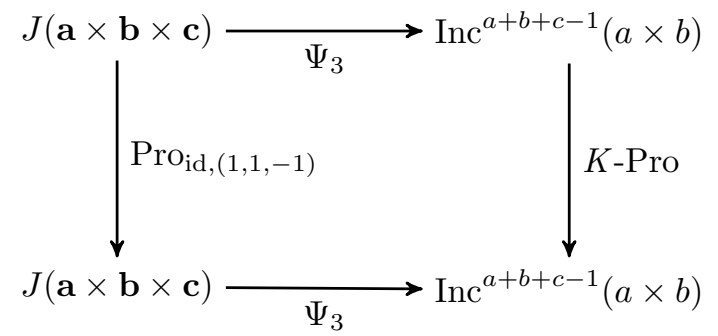

Proof: (sketch) One can check that composing the toggles for all elements lying on the hyperplane $\langle x,(1,1,-1)\rangle=(a-1)+(b-1)-\ell$ on the plane partition is equivalent to applying the $\ell$ th $K$ Bender-Knuth involution $K-\mathrm{BK}_{\ell}$ on the corresponding increasing tableaux. See [DPS16] for details.

Remark 4.3 By symmetry of $J(\mathbf{a} \times \mathbf{b} \times \mathbf{c})$, we obtain analogous results for $\Psi_{1}$ and $\Psi_{2}$.

As a consequence of the above lemma and Theorem 3.19, we obtain the following.

Theorem 4.4 $J(\mathbf{a} \times \mathbf{b} \times \mathbf{c})$ under Row is in equivariant bijection with $\operatorname{Inc}^{a+b+c-1}(a \times c)$ under K-Pro.

\subsection{Consequences and conjectures}

In this subsection, we give some consequences of Theorem 4.4 and some conjectures. The following is a consequence of Theorems 4.4 and 2.2 .

Corollary $4.5\left(J(\mathbf{a} \times \mathbf{b} \times \mathbf{c})\right.$, Row, Con $\left.\circ \Psi_{3}\right)$ exhibits resonance with frequency $a+b+c-1$.

We furthermore obtain the following corollary via the tri-fold symmetry of $J(\mathbf{a} \times \mathbf{b} \times \mathbf{c})$.

Corollary 4.6 There are K-Pro-equivariant bijections between $\operatorname{Inc}^{a+b+c-1}(a \times b)$, $\operatorname{Inc}^{a+b+c-1}(a \times c)$, and $\operatorname{Inc}^{a+b+c-1}(b \times c)$.

Proof: By Lemma 4.2 and Remark $4.3, \Psi_{2} \circ \Psi_{3}^{-1}$ is a $K$-Pro-equivariant bijection from $\operatorname{Inc}^{a+b+c-1}(a \times$ $b)$ to $\operatorname{Inc}^{a+b+c-1}(a \times c)$. Similarly, $\Psi_{1} \circ \Psi_{3}^{-1}$ is an equivariant bijection from $\operatorname{Inc}^{a+b+c-1}(a \times b)$ to $\operatorname{Inc}^{a+b+c-1}(b \times c)$.

Theorem 4.4 and Corollary 4.6 allows us to obtain a number of results for small values of $c$. We use the following trivial fact about the order of $K$-Pro on increasing tableaux of one row. 
Fact 4.7 The order of $K$-Pro on $\operatorname{Inc}^{q}(1 \times a)$ is $q$.

Corollary 4.8 The order of $K$-Pro on $\operatorname{Inc}^{a+b}(a \times b)$ is $a+b$.

Proof: By Corollary 4.6 there is a $K$-Pro-equivariant bijection between $\operatorname{Inc}^{a+b}(a \times b)$ and $\operatorname{Inc}^{a+b}(1 \times a)$. The result is then immediate by Fact 4.7

Corollary 4.9 The order of $K$-Pro on $\operatorname{Inc}^{a+b+1}(a \times b)$ is $a+b+1$.

Proof: By Corollary 4.6 , there is a $K$-Pro-equivariant bijection between the sets $\operatorname{Inc}^{a+b+1}(a \times b)$ and $\mathrm{Inc}^{a+b+1}(2 \times a)$. The result is then immediate from [Pe14, Theorem 1.3].

The following is a new proof of Theorem 3.1 of A. Brouwer-A. Schrijver [BrSc74], which states the order of Row on $J(\mathbf{a} \times \mathbf{b})$ is $a+b$.

Proof of Theorem 3.1: The order of Row on $J(\mathbf{a} \times \mathbf{b})$ is the same as the order of Row on $J(\mathbf{a} \times \mathbf{b} \times \mathbf{1})$. By Theorem 4.4 the order of Row on $J(\mathbf{a} \times \mathbf{b} \times \mathbf{1})$ equals the order of $K$-Pro on $\operatorname{Inc}^{a+b}(a \times 1)$. By Fact 4.7 the order of $K$-Pro on $\operatorname{Inc}^{a+b}(a \times 1)$ is $a+b$.

We can also use Theorem 4.4 and Corollary 4.6 to show that the Theorem 3.2 of P. Cameron-D. Fonder-Flaass [CaFo95] is equivalent to a theorem of the second author on increasing tableaux, thus providing a new proof of Theorem 3.2 .

Finally, we note an improvement on the bound in Theorem 3.3 of P. Cameron-D. Fon-der-Flaass [CaFo95] by more than a factor of $\frac{2}{3}$. This is evidence toward the conjecture of P. Cameron-D. Fon-der-Flaass [CaFo95] that this condition may be dropped entirely.

Theorem 4.10 If $a+b+c-1$ is prime and $c>\frac{2 a b-2}{3}-a-b+2$, then the cardinality of every orbit of Row on $J(\mathbf{a} \times \mathbf{b} \times \mathbf{c})$ is a multiple of $a+b+c-1$.

See [DPS16] for the proof of this theorem, which relies on showing that $K$-promotion acts cyclically not only on the content, but also on descents of the tableaux.

We conclude with a conjecture and some open problems.

Conjecture 4.11 The order of Row on $J(\mathbf{a} \times \mathbf{b} \times \mathbf{3})$ is $a+b+2$.

We have verified Conjecture 4.11 for $a+b \leq 11$. Finally, we reformulate some observations from [StWi12] as resonance conjectures; for further details, see [DPS16] and [StWi12, Sections 8.3 and 8.4].

Open Problem 4.12 Construct a natural map $f$ such that $\left(\mathrm{ASM}_{n},\langle\mathrm{SPro}\rangle, f\right)$ exhibits resonance with frequency $3 n-2$, where SPro is a certain toggle group action on the nth alternating sign matrix poset.

Open Problem 4.13 Construct a natural map $f$ such that $\left(\operatorname{TSSCPP}_{n},\langle\mathrm{Row}\rangle, f\right)$ exhibits resonance with frequency $3 n-2$, where $\mathrm{TSSCPP}_{n}$ is the set of totally symmetric self-complementary plane partitions inside a $2 n \times 2 n \times 2 n$ box.

\section{Acknowledgments}

These work began at the "Dynamical Algebraic Combinatorics" workshop at the American Institute of Mathematics (AIM) in March 2015. The authors are grateful for many inspiring conversations with the participants. The authors would like to thank Jim Propp, Tom Roby, and Nathan Williams for their organizational efforts, as well as AIM for funding this workshop and the AIM staff for their hospitality. 


\section{References}

[BeKn72] E. Bender and D. Knuth, Enumeration of plane partitions, J. Combin. Theory Ser. A 13 (1972), 40-54.

[BPS16] J. Bloom, O. Pechenik and D. Saracino, Proofs and generalizations of a homomesy conjecture of Propp and Roby, Discrete Math. 339 (2016), 194-206.

[BrSc74] A. Brouwer and A. Schrijver, On the period of an operator, defined on antichains, Math. Centrum report ZW 24/74 (1974).

[CaFo95] P. Cameron and D. Fon-der-Flaass, Orbits of antichains revisited, Eur. J. Combin. 16 (1995), 545-554.

[DPS16] K. Dilks, O. Pechenik, and J. Striker, Resonance in orbits of plane partitions and increasing tableaux, preprint, arXiv:1512.00365.

[HPPW16] Z. Hamaker, R. Patrias, O. Pechenik and N. Williams, Doppelgängers: Bijections of plane partitions, preprint, arXiv:1602.05535.

[Pe14] O. Pechenik, Cyclic sieving of increasing tableaux and small Schröder paths, J. Combin. Theory Ser. A 125 (2014), 357-378.

[PSV14] T. Pressey, A. Stokke and T. Visentin, Increasing tableaux and generalized Narayana numbers, to appear, Ann. Comb., arXiv:1409.2841.

[Pr83] R. Proctor, Shifted plane partitions of trapezoidal shape, Proc. Amer. Math. Soc. 89 (1983), 553-559.

[PrRo15] J. Propp and T. Roby, Homomesy in products of two chains, Electron. J. Combin. 22 (2015), 1-29.

[Pr01] J. Propp, The many faces of alternating-sign matrices, Discrete Math. Theor. Comput. Sci. Proc. AA (2001), 43-58.

[RSW04] V. Reiner, D. Stanton and D. White, The cyclic sieving phenomenon, J. Combin. Theory Ser. A 108 (2004), 17-50.

[Rh15] B. Rhoades, A skein action of the symmetric group on noncrossing partitions, preprint, arXiv:1501.04680.

[Sc72] M.-P. Schützenberger, Promotion des morphismes d'ensembles ordonnés, Discrete Math. 2 (1972), 73-94.

[StWi12] J. Striker and N. Williams, Promotion and rowmotion, Eur. J. Combin. 33 (2012), 19191942.

[ThYo09] H. Thomas and A. Yong, A jeu de taquin theory for increasing tableaux, with applications to K-theoretic Schubert calculus, Algebra Number Theory 3 (2009), 121-148.

[Wi00] B. Wieland, A large dihedral symmetry of the set of alternating sign matrices, Electron. J. Combin. 7 (2000). 